\title{
An improved bound on the minimal number of edges in color-critical graphs
}

\author{
Michael Krivelevich * \\ School of Mathematics, \\ Institute for Advanced Study, Princeton, NJ 08540, USA. \\ AMS Subject Classification: 05C15, 05C35. \\ Submitted: June 26, 1997. Accepted: November 24, 1997.
}

\begin{abstract}
It is proven that for $k \geq 4$ and $n>k$ every $k$-color-critical graph on $n$ vertices has at least $\left(\frac{k-1}{2}+\frac{k-3}{2\left(k^{2}-2 k-1\right)}\right) n$ edges, thus improving a result of Gallai from 1963 .
\end{abstract}

A graph $G$ is $k$-color-critical (or simply $k$-critical) if $\chi(G)=k$ but $\chi\left(G^{\prime}\right)<k$ for every proper subgraph $G^{\prime}$ of $G$, where $\chi(G)$ denotes the chromatic number of $G$. (See, e.g., [2] for a detailed account of graph coloring problems). Consider the following problem: given $k$ and $n$, what is the minimal number of edges in a $k$-critical graph on $n$ vertices? It is easy to see that every vertex of a $k$-critical graph $G$ has degree at least $k-1$, implying $|E(G)| \geq \frac{k-1}{2}|V(G)|$. Gallai [1] improved this trivial bound to $|E(G)| \geq\left(\frac{k-1}{2}+\frac{k-3}{2\left(k^{2}-3\right)}\right)|V(G)|$ for every $k$-critical graph $G$ (where $k \geq 4$ ), which is not a clique $K_{k}$ on $k$ vertices. In this note we strengthen Gallai's result by showing

Theorem 1 Suppose $k \geq 4$, and let $G=(V, E)$ be a $k$-critical graph on more than $k$ vertices. Then

$$
|E(G)| \geq\left(\frac{k-1}{2}+\frac{k-3}{2\left(k^{2}-2 k-1\right)}\right)|V(G)| .
$$

*e-mail: mkrivel@math.ias.edu 
THE ELECTRONiC JOURNAL OF COMBinAtorics 1 (1998), \#R4

In the first non-trivial case $k=4$ we get $|E(G)| \geq \frac{11}{7}|V(G)|$, compared to the estimate $|E(G)| \geq \frac{20}{13}|V(G)|$ of Gallai.

Let us introduce some definitions and notation (we follow the terminology of [4]). If $G=(V, E)$ is a $k$-critical graph, then the low-vertex subgraph of $G$, denoted by $L(G)$, is the subgraph of $G$, induced by all vertices of degree $k-1$. The high-vertex subgraph of $G$, which we denote by $H(G)$, is the subgraph of $G$ induced by all vertices of degree at least $k$ in $G$. Brooks' theorem implies that if $k \geq 4$ and $G \neq K_{k}$, then $H(G) \neq \emptyset$. A maximal by inclusion connected subgraph $B$ of a graph $G$ such that every two edges of $B$ are contained in a cycle of $G$ is called a block of $G$. A connected graph all of whose blocks are either complete graphs or odd cycles is called a Gallai tree, a Gallai forest is a graph all of whose connected components are Gallai trees. A $k$-Gallai forest (tree) is a Gallai forest (tree), in which all vertices have degree at most $k-1$.

Our proof utilizes results of Gallai [1] and Stiebitz [5], describing the structure of colorcritical graphs. Gallai proved the following fundamental result.

Lemma 1 ([1], Satz E.1) If $G$ is a k-critical graph then its low-vertex subgraph $L(G)$ is a $k$-Gallai forest (possibly empty).

Using induction on the number of vertices, it follows from the above statement that

Lemma 2 ([1], Lemma 4.5) Let $k \geq 4$. Let $G=(V, E) \neq K_{k}$ be a $k$-Gallai forest. Then

$$
|E(G)| \leq\left(\frac{k-2}{2}+\frac{1}{k-1}\right)|V(G)|-1 .
$$

The second ingredient of our proof is the following result of Stiebitz.

Lemma 3 ([5]) Let $G$ be a $k$-critical graph containing at least one vertex of degree $k-1$. Then the number of connected components of its high-vertex subgraph $H(G)$ does not exceed the number of connected components of its low-vertex subgraph $L(G)$.

Proof of Theorem 1. Let $L(G)$ and $H(G)$ be the low-vertex and the high-vertex subgraphs of $G$, respectively. Denote $n_{L}=|V(L(G))|, n_{H}=|V(H(G))|, n=|V(G)|=n_{L}+n_{H}$. By Brooks' theorem $n_{H}>0$. Also, if $V(L(G))=\emptyset$, we are done, therefore we may assume that $n_{L}>0$. 
THE EleCtronic Journal of COMBinatorics 1 (1998), \#R4

Let $r$ be the number of connected components of $H(G)$, then trivially

$$
|E(H(G))| \geq n_{H}-r
$$

Also, by Lemma 3, the number of connected components of $L(G)$ is at least $r$. Now the crucial observation is that each connected component of $L(G)$ is itself a $k$-Gallai tree, therefore the estimate (1) is valid for it too. We infer that

$$
|E(L(G))| \leq\left(\frac{k-2}{2}+\frac{1}{k-1}\right) n_{L}-r .
$$

Indeed, if $G_{1}=\left(V_{1}, E_{1}\right), \ldots, G_{r^{\prime}}=\left(V_{r^{\prime}}, E_{r^{\prime}}\right)$ are the connected components of $L\left(G^{\prime}\right)$, where $r^{\prime} \geq r$, then by Lemma 1

$$
\left|E_{i}\right| \leq\left(\frac{k-2}{2}+\frac{1}{k-1}\right)\left|V_{i}\right|-1, \quad i=1, \ldots, r^{\prime} .
$$

Summing the above inequalities over $1 \leq i \leq r^{\prime}$, we get $(3)$.

Using (2) and (3), the number of edges of $G$ can be bounded from below as follows:

$$
\begin{aligned}
|E(G)| & =\sum_{v \in V(L(G))} d(v)-|E(L(G))|+|E(H(G))| \\
& \geq(k-1) n_{L}-\left(\frac{k-2}{2}+\frac{1}{k-1}\right) n_{L}+r+n_{H}-r \\
& =n+\frac{k^{2}-3 k}{2(k-1)} n_{L} .
\end{aligned}
$$

On the other hand, it follows from the definition of $L(G)$ and $H(G)$ that

$$
\begin{aligned}
|E(G)| & =\frac{1}{2} \sum_{v \in V(G)} d(v)=\frac{1}{2}\left(\sum_{v \in V(L(G))} d(v)+\sum_{v \in V(H(G))} d(v)\right) \\
& \geq \frac{1}{2}\left((k-1) n_{L}+k n_{H}\right)=\frac{k}{2} n-\frac{1}{2} n_{L} .
\end{aligned}
$$

Multiplying (5) by $\left(k^{2}-3 k\right) /(k-1)$ and summing with (4) we get

$$
\left(1+\frac{k^{2}-3 k}{k-1}\right)|E(G)| \geq\left(1+\frac{k}{2} \frac{k^{2}-3 k}{k-1}\right) n
$$

or

$$
|E(G)| \geq\left(\frac{k-1}{2}+\frac{k-3}{2\left(k^{2}-2 k-1\right)}\right) n,
$$


THE ELECTRONiC JOURNAL OF COMBINATORICs 1 (1998), \#R4

as claimed.

A more detailed treatment of the problem, containing lower and upper bounds on the minimal number of edges in a $k$-critical graph on $n$ vertices with additional restrictions imposed, and some applications of these bounds to Ramsey-type problems and problems on random graphs, will appear in a forthcoming paper [3].

\section{References}

[1] T. Gallai, Kritische Graphen I, Publ. Math. Inst. Hungar. Acad. Sci. 8 (1963), 265-292.

[2] T. R. Jensen and B. Toft, Graph coloring problems, Wiley, New York, 1995.

[3] M. Krivelevich, On the minimal number of edges in color-critical graphs, Combinatorica, to appear.

[4] H. Sachs and M. Stiebitz, Colour-critical graphs with vertices of low valency, Annals of Discrete Math. 41 (1989), 371-396.

[5] M. Stiebitz, Proof of a conjecture of T. Gallai concerning connectivity properties of colour-critical graphs, Combinatorica 2 (1982), 315-323. 